\title{
INFLUÊNCIA DOS MODOS DE VARIABILIDADE CLIMÁTICA SOBRE EVENTOS DE RESSACAS NO LITORAL SUL DO ESPÍRITO SANTO, BRASIL
}

\author{
EGUCHI, Branco - bmeguchi@hotmail.com \\ Universidade Federal do Espírito Santo / UFES \\ ALBINO, Jacqueline - albino.jacqueline@gmail.com \\ Universidade Federal do Espírito Santo / UFES
}

Submetido em: 01/04/2020

Aceito para publicação em: 14/12/2020

Publicado em: 06/04/2021

DOI: http://dx.doi.org/10.5380/abclima.v28i0.72614

\begin{abstract}
RESUMO: A partir de dados de reanálise de ondas entre os anos de 1948 e 2008 do modelo GOW e dos índices El Niño Oscilação Sul (ENSO) e Modo Anular Sul (SAM), obtidos através da Administração nacional oceânica e atmosférica dos EUA (National Oceanographic and Atmospheric Administration - NOAA), descreveu-se o clima de ressacas marinhas e a relação dos modos ENSO e SAM com os padrões associados à energia e ao número das ressacas no litoral sul do estado de Espírito Santo. As ressacas foram definidas como eventos com alturas de ondas superiores à média dos $5 \%$ das ondas mais altas do banco dados $\left(\mathrm{H}_{5 \%}\right)$ e duração mínima de 24 horas. Os resultados apontaram 208 eventos de ressacas entre 1948 e 2008, com altura limite mínima para ondas $\left(\mathrm{H}_{5 \%}\right)$ de $1,72 \mathrm{~m}$, duração média de 2 dias e direção preferencial de sul/sudeste. No entanto, as ressacas com maiores energias acumuladas foram provenientes do quadrante leste e possuíram duração máxima de 7 dias. O número de ressacas e a energia acumulada/duração apresentaram incremento a partir de março, atingindo valores máximos em setembro e agosto, respectivamente. Assim, a temporada de ressacas no litoral sul do Espírito tem seu auge entre os meses de julho e setembro e é caracterizada por eventos duradouros e energéticos que evoluem para eventos breves e mais frequentes. Durante os 60 anos analisados, $52 \%$ dos eventos de ENSO foram neutros, $25 \%$ positivos e $23 \%$ negativos. A variabilidade do SAM apresentou uma maior ocorrência da fase negativa (66\%) quando comparada a fase positiva (34\%). O teste Chi-Quadrado de Pearson, para um nível de confiança de $95 \%$, apontou que energia, duração e a quantidade de ressacas são estatisticamente dependentes das fases dos modos climáticos isolados e combinados, com exceção da duração e quantidade de ressacas em relação as fases do SAM. Durante a fase negativa do SAM a energia das ressacas foi maior quando comparada a fase positiva. Para o ENSO, todos os parâmetros apresentaram maiores valores durante a fase neutra e menores valores durante a fase positiva. Apesar de a fase neutra do ENSO ocorrer duas vezes mais do que a fase negativa, o valor acumulado de energia, duração e quantidade foi apenas 0,5 vezes maior, evidenciando o alto potencial de ressacas na fase negativa do ENSO. Ao considerar as combinações entre as fases dos modos, observou-se que a combinação SAM positivo e ENSO neutro apresentou os maiores valores para todos os parâmetros de ressacas. O estudo ressalta a importância da atuação conjunta dos modos de variabilidade climática sobre o clima de ressacas.
\end{abstract}

PALAVRAS-CHAVE: Modelo Global Ocean Wave, Anticiclone Subtropical do Atlântico Sul, Modo Anular Sul, El Niño Oscilação Sul, Tempestades

CLIMATIC VARIABILITY MODES EFFECTS ON THE STORM WAVES OCCURRENCE ON THE SOUTHERN COAST OF ESPÍRITO SANTO, BRAZIL

ABSTRACT: Based on wave data between 1948 and 2008 from GOW model reanalysis, El Niño Southern Oscillation (ENSO) and South Annular Mode (SAM) indexes, obtained from the National Oceanic and Atmospheric Administration (NOAA), the climate of storm 
waves and the relationship with ENSO and SAM modes, associated with energy and amount of storms was described at the southern coast of the state of Espirito Santo. Storm waves were defined as events with wave heights above the mean of the highest $5 \%$ waves in the database $(\mathrm{H} 5 \%)$ and minimum duration of 24 hours. The results indicated 208 events of storm waves between 1948 and 2008, with minimum wave height ( $\mathrm{H} 5 \%$ ) of $1.72 \mathrm{~m}$, from south/southeast and average duration of 2 days. However, events with the highest accumulated wave energy were from east and lasted for 7 days. The amount and accumulated energy/duration of storm waves increased from March, reaching maximum values in September and August, respectively. Thus, the season of storm waves on the southern coast of Espírito show its peak between July and September and is characterized by lasting and energetic events that evolve to brief and more frequent events. During the 60 years analyzed, 52\% of the ENSO events were neutral, $25 \%$ positive and $23 \%$ negative. The variability of the SAM showed a greater occurrence during the negative phase $(66 \%)$ when compared with the positive phase $(34 \%)$. Pearson's Chi-Square test, for a 95\% confidence level, showed that energy, duration and the amount of storm waves are statistically dependent on the phases of the climate modes alone and combined, except for the duration and amount related to SAM phases. During the negative phase of SAM, the energy of storm waves was higher when compared to the positive phase. For ENSO, all parameters showed greater values during the neutral phase and lower values during the positive phase. Although the neutral phase of ENSO occurs twice as much as the negative phase, the accumulated value of energy, duration and amount was only 0.5 times greater, highlighting the storm wave potential during the negative phase of ENSO. When considering the combinations between the modes phases, it was observed that the combination of positive SAM and neutral ENSO presented the greatest values for all parameters of storm waves. Thus, the study highlights the importance of combined action of modes of climate variability on the climate of storm waves

KEYWORDS: Global Ocean Wave model, South Atlantic Anticyclone, Southern Annular Mode, El Niño Southern Oscillation, Storms.

\section{EFECTOS DE LOS MODOS DE VARIABILIDAD CLIMÁTICA EN LA OCURRENCIA DE RESACA MARINA EN LA COSTA SUR DE ESPÍRITO SANTO, BRASIL}

RESUMEN: Basado en datos de reanálisis de olas entre 1948 y 2008 del modelo GOW y los índices de Oscilación Sur de El Niño (ENSO) y Modo Anular Sur (SAM), obtenido de la Administración Nacional Oceánica y Atmosférica (National Oceanographic and Atmospheric Administration - NOAA), se describió el clima de olas de tormenta y la relación con los modos ENSO y SAM, con los patrones asociados con la energía y el número de tormentas en la costa sur del estado de Espírito Santo. Las olas de tormenta se definieron como eventos con alturas de ola más grande que de la media del $5 \%$ de las olas más altas en la base de datos (H5\%) y duración mínima de 24 horas. Los resultados indicaron 208 eventos de olas de tormenta entre 1948 y 2008, con límite mínimo de altura $(\mathrm{H} 5 \%)$ de $1,72 \mathrm{~m}$, desde el sur/sureste y una duración media de 2 días. Sin embargo, las olas de tormenta con las energías acumuladas más altas provenían del este y tenían una duración máxima de 7 días. El número y la energía/duración acumulada de las olas de tormenta aumentaron a partir de marzo, alcanzando los valores máximos en septiembre y agosto, respectivamente. Así, la temporada de olas de tormenta en la costa sur de Espírito muestra su apogeo entre julio y septiembre y se caracteriza por eventos duraderos y energéticos que evolucionan a eventos breves y más frecuentes. Durante los 60 años analizados, $52 \%$ de los eventos del ENSO fueron neutrales, $25 \%$ positivos y $23 \%$ negativos. La variabilidad del SAM mostró una mayor ocurrencia durante la fase negativa (66\%) en comparación con la fase positiva (34\%). La prueba Chi-Cuadrado de Pearson, para un nivel de confianza del 95\%, mostró que la energía, la duración y el número de olas de tormenta dependen estadísticamente de las fases de los modos climáticos solos y combinados, excepto por la duración y el número relacionados con las fases del SAM. Durante la fase negativa del SAM, la energía de las olas de tormenta fue mayor en comparación con la fase positiva. Para ENSO, todos los parámetros mostraron 
valores mayores durante la fase neutra y valores inferiores durante la fase positiva. Aunque la fase neutra de ENSO ocurre dos veces más que la fase negativa, el valor acumulado de energía, duración y número fue sólo 0,5 veces mayor, destacando el potencial de las olas de tormenta durante la fase negativa de ENSO. Al considerar las combinaciones entre las fases de modos, se observó que la combinación de SAM positivo y ENSO neutral presentaba los mayores valores para todos los parámetros. Así, el estudio destaca la importancia de la acción combinada de los modos de variabilidad climática en el clima de las olas de tormenta.

PALABRAS ClAVE: Modelo Global Ocean Wave, Anticiclón Subtropical del Atlántico sur, Modo de Anillo sur, El Niño oscilación sur, Tormentas

\section{INTRODUÇÃO}

Innocentini et al. (2003) afirmam que a agitação marítima de maior intensidade no Atlântico Sul está associada a passagem de frentes frias relacionadas a ciclones que se desenvolvem entre as latitudes de $20^{\circ} \mathrm{S}$ e $57,5^{\circ} \mathrm{S}$ (PARISE, 2009; REBOITA ET AL., 2005), migram no sentido SO-NE e são caracterizados por ventos do quadrante sul/sudeste na costa brasileira (SATYAMURTY E MATTOS, 1989). A trajetória e intensidade dessas frentes frias são influenciadas por instabilidades baroclínicas (CARLSON, 1991; GAN E RAO, 1991; VERA ET AL., 2002), fluxos de quantidade de movimento e calor, ondas planetárias estacionárias (CHANG ET AL., 2002), intensidade do jato subtropical, cristas e cavados, subsidência em $500 \mathrm{hPa}$, gradientes de pressão entre continente e oceano, intensidade dos ventos na região de confluência sobre o oceano e advecção de vorticidade positiva (CAVALCANTI E KUOSKY, 2009). Analisar o comportamento das frentes frias ao longo da costa do Brasil requer uma avaliação da variabilidade do Anticiclone ou Alta Pressão Subtropical do Atlântico Sul (ASAS), já que este é o controle principal da circulação da bacia do oceano Atlântico Sul (LÜBBECKE ET AL., 2014; SUN ET AL., 2017) e sua posição determina a intensidade do bloqueio na migração de ciclones em direção ao equador (DA SILVEIRA PEREIRA E KLUMB-OLIVEIRA, 2015). Segundo Sun et al. (2017), o ciclo anual do ASAS mostra dois picos de intensidade e tamanho, que ocorrem durante os meses de solstício. Durante os meses de equinócio o ASAS enfraquece e se contrai. No verão o ASAS é deslocado para sul, com seu centro no meio da bacia do oceano Atlântico, já durante o inverno o centro do ASAS se encontra mais próximo da linha do equador. O ASAS também apresenta variações associadas aos modos de variabilidade climática, como por exemplo Oscilação do Atlântico Norte (NAO), Padrão Pacífico-América do Norte (PNA) (VENEGAS ET AL., 1997) e, em especial, El Niño Oscilação Sul (El Niño Southern Oscillation -ENSO) (DA SILVEIRA PEREIRA E KLUMB-OLIVEIRA, 2015) e ao Modo Anular Sul ou Southern Annular Oscilation (SAM) (MARSHALL ET AL., 2018).

Segundo Kousky et al. (1984), durante a fase positiva do ENSO ocorre o fortalecimento do ASAS devido à intensificação da subsidência de ar nas regiões tropicais no Atlântico Sul. Este processo é desencadeado pela redução de pressão sobre o Oceano Pacífico, que aumenta a ascensão de ar e modifica a circulação zonal da Célula de Walker. O fortalecimento do ASAS intensifica os ventos alísios no Brasil e ocasiona a obstrução de frentes frias (ARAGÃO, 1998). Já na fase negativa do ENSO, ocorre o processo inverso e leva ao enfraquecimento do ASAS e dos ventos alísios. Desta forma, as frentes frias provenientes da região polar conseguem avançar com maior facilidade rumo a latitudes menores (RAO ET AL., 2002; PEZZA E AMBRIZZI, 2003; SILVA ET AL., 2009). 
De acordo com Lorenz e Hartmann (2001), o SAM é gerado pelo feedback positivo entre anomalias das médias zonais de velocidade do vento e fluxos de momento turbulento. Variações do SAM resultam de atividades anômalas de ondas baroclínicas em áreas de passagem de tempestades extratropicais em associação com as oscilações dos Jatos Polares (MARSHALL ET AL., 2018). Durante a fase positiva do SAM, os ventos de oeste e a baroclinicidade aumentam nas altas latitudes e diminuem próximo ao centro do cinturão do Jato Polar. Desta forma, a região de vórtices mais próxima ao polo se desloca para sul de sua posição climatológica média no hemisfério sul e traz consigo o limite sul da Célula de Hadley e toda a Célula de Ferrel (THOMPSON E WALLACE, 2000). Por outro lado, na fase negativa do SAM ocorrem o deslocamento dos ventos de oeste em direção ao equador e a redução da atividade de ondas baroclínicas na região de tempestades extratropicais (MARSHALL ET AL., 2018). Segundo Reboita et al. (2009), a fase positiva/negativa do SAM apresenta maior/menor concentração de ciclones ao redor do continente antártico e condições desfavoráveis/favoráveis para ciclogêneses em torno de $45^{\circ} \mathrm{S}$. Visto o impacto de cada modo sobre o padrão de circulação atmosférica, a atuação conjunta de ENSO e SAM pode resultar em configurações atmosféricas que propiciam (ENSO e SAM negativos) ou não (ENSO e SAM positivos) a formação de ressacas no litoral sudeste do Brasil.

Como efeito da passagem de frente frias tem-se o fenômeno das ressacas, caracterizadas por ondas provindas do quadrante sul, duração média de 2 dias e potencial de gerar danos à costa (BULHÕES ET AL., 2014; DA SILVEIRA PEREIRA E KLUMB-OLIVEIRA, 2015; DE SOUZA ET AL., 2016; LINSDE-BARROS ET AL., 2018). A caracterização das ressacas é feita a partir da análise da energia de onda, duração e número de eventos (YOU E LORD, 2008; MENDOZA E JIMÉNEZ, 2009; DURÁN ET AL., 2016; CASTELLE ET AL., 2017), fatores que determinam a severidade do evento (DISSANAYAKE ET AL., 2015; RAHMSTORF, 2017; GODOI ET AL., 2017). Godoi et al. (2014) concluem que as maiores alturas de ondas ocorrem durante o inverno, associadas ao padrão de passagens de frentes frias. Da Silveira Pereira e Klumb-Oliveira (2015) avaliaram o impacto do ENSO sobre as alturas significativas de ondas no Sudeste e concluíram que há uma ligeira redução de alturas de ondas durante a fase positiva do ENSO e o inverso para a fase negativa. Pianca et al. (2010) ressaltam a escassez de informações e estudos que envolvem medições de ondas no Brasil, no entanto, apontam o desenvolvimento de modelos numéricos como uma opção para a caracterização do clima de ondas. Dada a importância dos efeitos dos modos climáticos sobre as alturas de ondas e os potenciais impactos costeiros relacionados aos eventos de ressacas, o presente estudo objetiva realizar uma avaliação da influência isolada e combinada dos modos de variabilidade climática sobre as ressacas, associadas a frentes frias, nas escalas sazonal e mensal para o litoral sul do estado de Espírito Santo.

\section{MÉTODOS}

\section{BANCO DE DADOS GOW}

O banco de dados de ondas utilizado representa a média diária de dados oriundos do modelo Global Ocean Wave (GOW), disponibilizado pelo programa SMC-Brasil (IH-Cantábria). O modelo consiste em uma reanálise global de dados de ondas (REGUERO ET AL. 2012) e fornece alturas significativas, direções e 
períodos de picos entre 1948 e 2008 para um grid com resolução espacial de $1,5^{\circ}$ de longitude e $1^{\circ}$ de latitude, cuja posição central é $21^{\circ} \mathrm{S}, 40^{\circ} 30^{\prime} \mathrm{O}$ (Figura 1). O grid foi recortado do modelo global e se localiza adjacente ao litoral sul do estado do Espírito Santo, cujo padrão de ventos apresenta comportamento bimodal, com ventos de leste/nordeste gerados pelo ASAS, predominantes durante o verão, e fortes ventos do quadrante sul/sudeste, originados de frentes frias polares durante os meses de inverno (AMARANTE ET AL., 2009). Nogueira et al. (2015) definem, através de simulações com o modelo WAVEWATCH III, três principais categorias de estado de mar com base nos sistemas meteorológicos que dominam a Bacia do Espírito Santo: a) Bom Tempo, com ondas de E e NE associadas ao ASAS; b) Bom Tempo com swell, que representa a condição de Bom Tempo com ondulações ou swell distante e c) Mau Tempo, com ondas de S e SE associadas aos sistemas frontais. Albino et al. (2016a) apresentam a climatologia de ondas do litoral capixaba com alturas de ondas médias variando entre 1 e 1,5 m provenientes de leste, com base em dados de reanálise do banco de dados do modelo GOW entre os anos de 1948 e 2008 a uma profundidade de $30 \mathrm{~m}$. O litoral é caracterizado por um regime de micromaré, com preamar e baixamar de sizígia que atingem $1,8 \mathrm{~m}$ e $-0,2 \mathrm{~m}$, respectivamente (DHN, 2020).
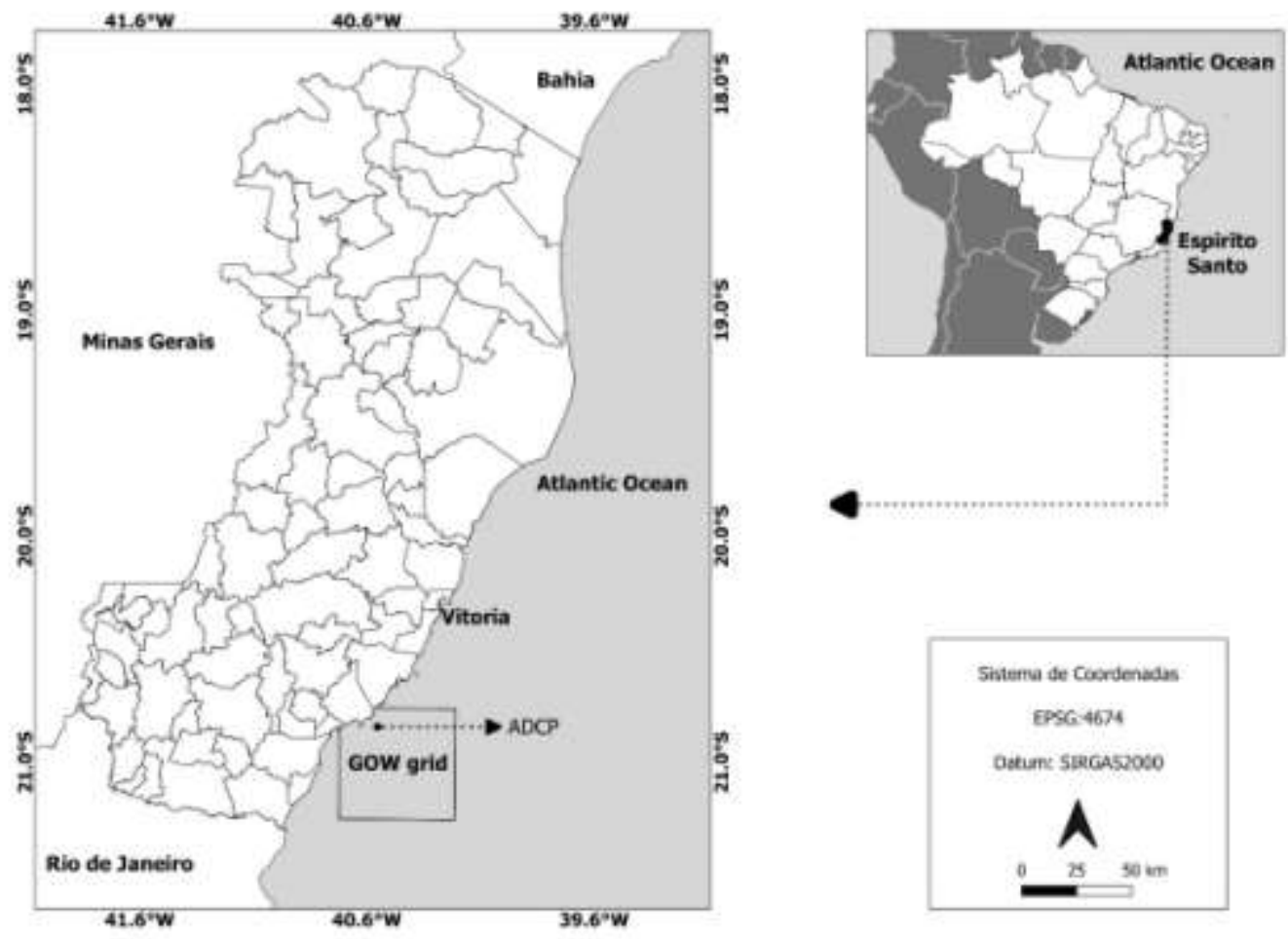

Figura 1 - Mapa de localização do estado do Espírito Santo e do grid de dados de ondas do modelo GOW.

Albino et al. (2016b) compararam os dados utilizados neste estudo com dados de ondas medidos por um Acoustic Doppler Current Profiler (ADCP) no ponto $20^{\circ} 48^{\prime} \mathrm{S}, 40^{\circ} 33^{\prime} \mathrm{O}$ (Figura 1 ), a uma profundidade de $25 \mathrm{~m}$ durante os períodos de a)31/01/2007 a 04/04/2007, b)29/09/2007 a 25/10/2007, c) $23 / 11 / 2007$ a $18 / 12 / 2007$ e d)23/11/2007 a 06/03/2008. Com base no cálculo de viés e Raiz do Erro Quadrático Médio (Root Mean Square Error - 
RMSE), os autores afirmam que o modelo apresenta boa acurácia na evolução e magnitude da altura significativa, direção e período de pico de onda. No entanto, o efeito de ondas geradas por ventos locais causa variações episódicas de menos de $45^{\circ}$ e 6 s na direção e período de pico, respectivamente. A Tabela 1 traz as informações estatísticas de viés e RSME da comparação da altura significativa, período e direção de pico para cada intervalo.

Tabela 1 - Parâmetros estatísticos de comparação dos dados de ondas medidos e modelados. Modificado de Albino et al. (2016b).

\begin{tabular}{|c|c|c|c|c|c|c|}
\hline \multirow[t]{2}{*}{ Intervalo } & \multicolumn{2}{|l|}{$\mathrm{H}_{3}(\mathrm{~m})$} & \multicolumn{2}{|l|}{$T_{p}(s)$} & \multicolumn{2}{|l|}{$\theta\left({ }^{*}\right)$} \\
\hline & Vies & RMSE & Viè & RMSE & Vies & RMSE \\
\hline a) & 0.03 & 0.16 & $-0,33$ & 1.93 & 12.95 & 41.52 \\
\hline b) & 0.02 & 0.17 & 0.02 & 1.55 & 7.51 & 19.38 \\
\hline c) & -0.02 & 0.23 & 0.09 & 1.34 & 1279 & 26.12 \\
\hline d) & 0.14 & 024 & 0.22 & 1.03 & 13.44 & 28.60 \\
\hline
\end{tabular}

$\mathrm{Na}$ definição de ressacas adotou-se uma adaptação da metodologia baseada em You e Lord (2008), Durán et al. (2016), Earlie et al. (2017) e Castelle et al. (2017). Os estudos sugerem como limite ondas maiores ou igual a média dos $5 \%$ das ondas mais altas do banco de dados $\left(H_{5 \%}\right)$ e duração mínima de 12 horas. Neste trabalho considerou-se a duração mínima de 24 horas sugerido por Cowell e Thom (1994), limite que engloba processos com escala temporal suficiente para alterar a morfodinâmica local, como exemplo as tempestades e consequentes ressacas com potencial para promover 0 transporte sedimentar da porção emersa para a zona submersa da praia (MASSELINK E HUGHES, 2014). A intensidade do evento é determinada através do somatório da energia de onda, calculada através da teoria linear de ondas (HOLTHUIJSEN, 2007) (Equação 1).

$$
\sum_{i=1}^{n} E_{i}=\frac{1}{8} \rho g H_{i}^{2}
$$

Onde $E_{i}$ representa a energia por unidade, $\rho$ a densidade da água do mar, $H_{i}$ a altura da onda e $i$ cada dia em que se caracteriza ressaca.

Para determinação de diferenças significativas das características das ressacas entre as estações do ano foram realizados testes não paramétricos através do software livre Paleontological Statistics (PAST 3.6), disponibilizado pela Universidade de Oslo (HAMMER et al., 2001). O primeiro teste foi de Kruskal-Wallis (Equação 2 e Equação 3) (HAMMER, 2012), considerado uma extensão do teste Mann-Whitney para comparar diversos grupos (ZAR, 1996). Posteriormente, rejeitada a hipótese de que as medianas dos grupos são iguais, se realizou o teste pos hoc de Dunn, com intuito de comparar em pares os resultados e identificar a estações com comportamento de ressacas similares.

$$
H=\frac{12}{n(n+1)}\left(\sum_{g} \frac{T_{g}{ }^{2}}{n_{g}}\right)-3(n+1)
$$

Sendo $\mathrm{H}$ a estatística calculada, $\mathrm{n}_{\mathrm{g}}$ o número de elemento do grupo $\mathrm{g}, \mathrm{n}$ a quantidade total de elementos e $\mathrm{T}_{\mathrm{g}}$ a soma de ranks no grupo $\mathrm{g}$. 


$$
H_{c}=\frac{H}{1-\frac{\sum_{i} f_{i}^{3}-f_{i}}{n^{3}-n}}
$$

Onde $H_{c}$ representa o ajuste para valores repetidos, $F_{i}$ é a quantidade de elementos no grupo i de elementos repetidos. $O$ valor-p, que representa a semelhança $(p>=0,05)$ ou diferença $(p<0,05)$ estatística, é aproximado a partir de $\mathrm{H}_{\mathrm{c}}$ por meio da distribuição de qui-quadrado com Número de grupos-1 graus de liberdade.

\section{MODOS DE VARIABILIDADE CLIMÁTICAS}

Os modos climáticos escolhidos foram El Niño Oscilação Sul (ENSO) e Modo Anular do Sul (SAM), cujas informações foram retiradas do banco de dados disponibilizado pela Administração nacional oceânica e atmosférica dos EUA (National Oceanographic and Atmospheric Administration - NOAA), no formato trimestral (ENSO) e mensal (SAM) entre os anos de 1948 e 2008. Para que os índices possuíssem a mesma resolução temporal, os dados foram agrupados em médias por estação, de acordo com a seguinte divisão: verão (Dezembro, Janeiro e Fevereiro - DJF), outono (Março, Abril e Maio - MAM), inverno (Junho Julho e Agosto - JJA) e primavera (Setembro, Outubro e Novembro - SON).

Os índices de anomalias do ENSO representam o Índice Niño Oceânico (Oceanic Niño Index - ONI), calculados com base na média móvel da anomalia de temperatura a cada três meses obtida através da análise da base de dados Extended Reconstructed Sea Surface Temperature (ERSST. v5) para a região Niño 3.4 ( $5^{\circ} \mathrm{N}-5^{\circ} \mathrm{S}, 120 \mathrm{~W}-170^{\circ} \mathrm{W}$ ). Os dados do ERSST v5 são modelados em uma grade global de $2^{\circ} \times 2^{\circ}$, utilizando como input dados de flutuadores Argo proveniente do International Comprehensive Ocean-Atmosphere Dataset (ICOADS) (Huang et al., 2017). Por definição da NOAA, a fase positiva do ENSO (El Niño) ocorre quando o valor do índice ONI é superior e 0,5, assim como a fase negativas ( $L a$ Niña) se dá para valores menores do que -0,5; entre estes limites tem-se um ENSO neutro (LARKING E HARRISON, 2005).

Os índices do SAM são calculados a partir de dados de pressão atmosférica no nível do mar (SLP) pelo projeto de reanálise 20CRV2c, que consiste na reconstituição histórica em quatro dimensões dos padrões climáticos globais. O 20CRv2c funciona a partir da assimilação de dados através de filtragem que estima o estado mais provável da atmosfera a cada três horas e calcula as incertezas associadas. O cálculo do índice SAM é obtido através da diferença da média zonal da SLP entre $65^{\circ} \mathrm{S}$ e $40^{\circ} \mathrm{S}$. Neste sentido, é realizada a normalização dos valores mensais, dividindo as médias mensais pelo desvio padrão climatológico calculado entre os anos de 1981 à 2010 (PSD, 2019).

Posteriormente, foi realizada a quantificação de ocorrências de ressacas para cada fase do ENSO e SAM, considerando uma escala multidecadal (1948 a 2008) e sazonal, segundo Cardozo et al. (2015). Esta abordagem permite identificar a possível relação entre os modos climáticos e as ressacas. Em complemento, foram calculadas as médias de Duração, Energia de onda e Quantidade de ressacas ocorridas em cada fase do ENSO e SAM.

O teste Qui-Quadrado ( $\mathrm{Chi}^{2}$ ) de independência foi realizado para verificar se há dependência entre a os parâmetros das ressacas e as fases dos modos de 
maneira isolada e combinada. O cálculo dos valores de $\mathrm{Chi}^{2}$ foi realizado a partir dos acumulados de energia, duração e quantidade de ressacas para cada fase dos modos segundo a Equação 4.

$$
C h i^{2}=\sum_{i} \frac{\left(A c_{i}-F e\right)^{2}}{F e}
$$

Onde $A c_{i}$ é o valor acumulado para cada fase do modo climático e Fe representa a frequência esperada. O teste aceita a hipótese nula de que há independência entre os parâmetros de ressaca e as fases dos modos climáticos caso o valor de $\mathrm{Chi}^{2}$ calculado seja inferior ao $\mathrm{Chi}^{2}$ crítico tabelado para $(\mathrm{K}-1)$ graus de liberdade, onde $\mathrm{K}$ é o número de grupos em consideração (GOTELLI E ELLISON, 2016). O nível de confiança adotado neste estudo foi de $95 \%$.

\section{RESULTADOS}

\section{CARACTERIZAÇÃO DAS RESSACAS}

Foram identificados 208 eventos de ressacas entre 1948 e 2008, com altura limite mínima para ondas $\left(H_{5 \%}\right)$ de 1,72 m e duração mínima de 24 horas.

A

Figura 2 mostra que as ondas de ressacas foram preferencialmente provenientes do quadrante sudeste com alturas significativas inferiores a 2,5 m e períodos de pico entre 7 e 9 s, cuja duração média foi de 48 horas. Contudo, foram as ressacas de leste, com duração de até 7 dias e altura significativa média superior a $2,5 \mathrm{~m}$, que apresentaram os mais altos valores de energia acumulada de onda. Além disso, houve um incremento da energia acumulada relacionada ao aumento da duração da ressaca (Figura 3).
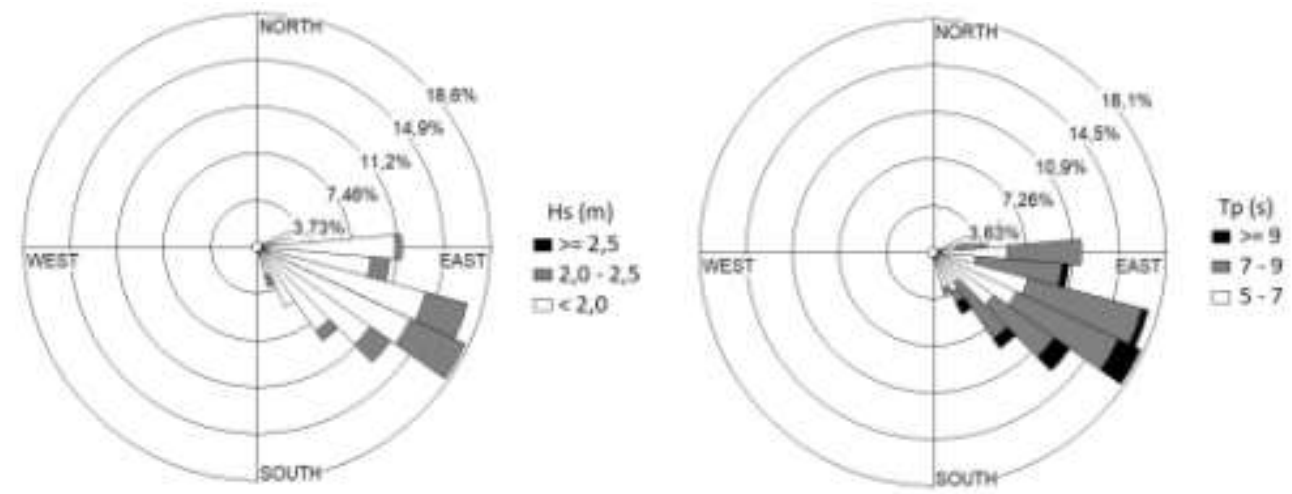

Figura 2 - Histograma direcional da distribuição de altura significativa (esquerda) e período de pico (direita) das ressacas identificadas no banco de dados GOW entre 1948 e 2008. 


\section{Direção e Duração x Energia}

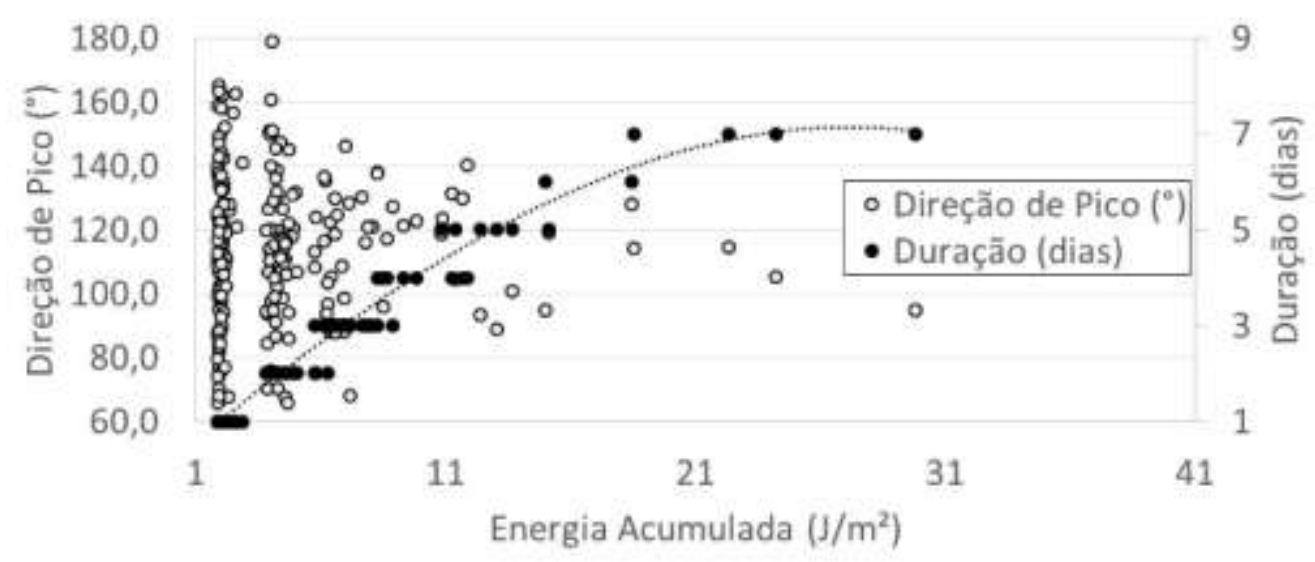

Figura 3 - Gráfico de dispersão entre direção de pico e energia de onda acumulada em cada ressaca (pontos sem preenchimento) e dispersão entre duração das ressacas e sua energia de onda acumulada (pontos preenchidos), com linha de tendência do tipo polinomial de ordem 3 (linha pontilhada).

Das 208 ressacas identificadas, 7\% ocorreram durante o verão (dez-janfev), $15 \%$ nos meses de outono (mar-abr-mai), 38\% no inverno (jun-jul-ago) e $40 \%$ na primavera (Set-Out-Nov) (Figura 4). Nota-se um aumento do número de ressacas e energia acumulada de março até setembro, mês com o maior valor acumulado de ressacas (41 eventos). Os maiores registros de ressaca e energia acumulada ocorreram nos meses de julho, agosto e setembro (inverno e primavera). O maior acumulado de energia de onda foi encontrado no inverno, com destaque para o mês de agosto. Na continuidade da primavera, nos meses de outubro e novembro, houve o decaimento no número de ressacas, contudo, os registros se mantiveram altos quando comparados aos valores do verão e outono.

O teste de Kruskal-Wallis aponta diferenças significativas entre as estações do ano, com valores de $\mathrm{p}<0,05$ para a energia acumulada, número e duração das ressacas (Tabela 2). Ao aprofundar a análise, o teste pos-hoc de Dunn mostra que outono e verão possuem características semelhantes com valor-p variando entre 0,06 (energia de onda e duração) e 0,08 (número de ressacas). Já o inverno se assemelha com a primavera com valor-p de 0,3 (energia de onda), 0,35 (duração) e 0,74 (número de ressacas) (Tabela 2). Apesar de não haver diferença estatística, quantitativamente no inverno as tempestades ocorreram em menor número, porém com maior energia acumulada quando comparadas às ocorrências da primavera, conforme ilustra a Figura 4. Isto se deve a maior duração das ressacas de inverno, um total de 79 ressacas com duração média de 72 horas, enquanto na primavera ocorrem 84 eventos com duração média de $62 \mathrm{~h}$. 


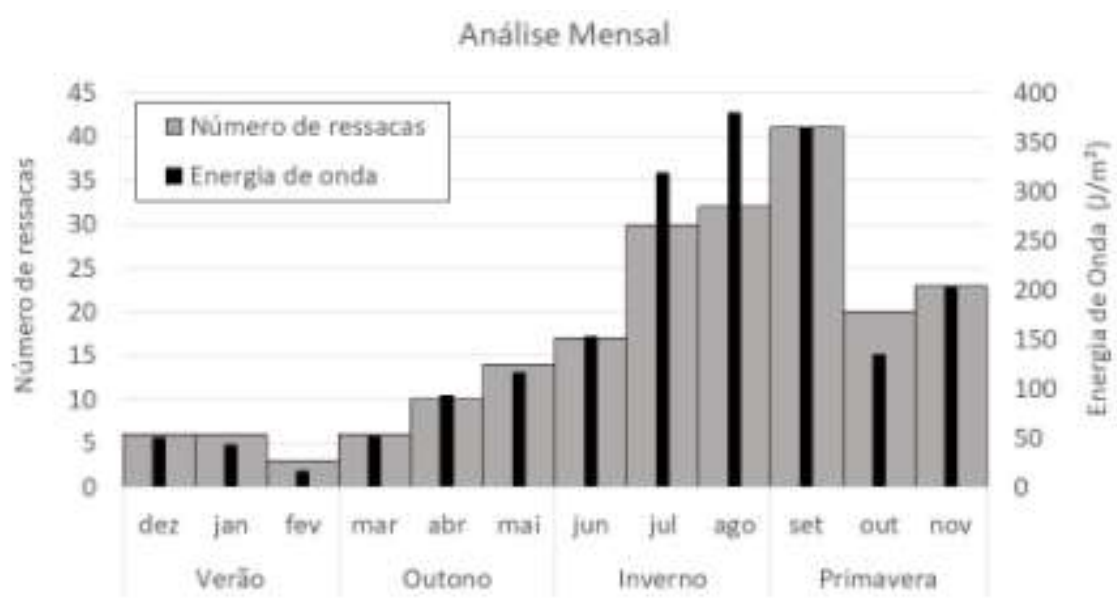

Figura 4 - Gráfico de colunas da quantificação do número de ressacas (cinza) e da energia de onda acumulada (preto) para cada mês durante os 60 anos de dados do banco de dados GOW.

Tabela 2 - Resumo dos testes não paramétricos de Kruskall-Wallis e pos hoc de Dunn realizados para compara os parâmetros de energia de onda, duração e número de ressacas entre as estações do ano.

Kruskal-Wallis

\begin{tabular}{|c|c|c|}
\hline Energia de Onda & Duração & Número de ressacas \\
\hline $\mathrm{H}=43$ & $\mathrm{H}=44$ & $\mathrm{H}=43$ \\
\hline $\mathrm{Hc}=49$ & $\mathrm{Hc}=51$ & $\mathrm{Hc}=51$ \\
\hline$p=0,00$ & $p=0,00$ & $p=0,00$ \\
\hline
\end{tabular}

pos hoc de Dunn

\begin{tabular}{|c|c|c|c|c|c|c|c|c|c|}
\hline & \multicolumn{3}{|c|}{ Energia de Onda } & \multicolumn{3}{|c|}{ Duração } & \multicolumn{3}{|c|}{ Número de ressacas } \\
\hline & Verão & Outono & Inverno & Verão & Outono & Inverno & Verão & Outono & Inverno \\
\hline Outono & 0,06 & - & - & 0,06 & - & - & 0,08 & - & - \\
\hline Inverno & 0,00 & 0,00 & - & 0,00 & 0,00 & - & 0,00 & 0,00 & - \\
\hline Primavera & 0,00 & 0,00 & 0,30 & 0,00 & 0,00 & 0,35 & 0,00 & 0,00 & 0,74 \\
\hline
\end{tabular}

\section{MODOS DE VARIABILIDADE CLIMÁTICA E DISTRIBUIÇÃO DAS RESSACAS}

A Figura 5 apresenta a variabilidade das fases de ENSO e SAM ao longo do período analisado. Durante os 60 anos, 52\% dos eventos de ENSO foram neutros, $25 \%$ positivos e $23 \%$ negativos. A variabilidade do SAM apresentou uma maior ocorrência da fase negativa (66\%) quando comparada a fase positiva (34\%).

A tabela 3 mostra os valores de $\mathrm{Chi}^{2}$ calculados para as observações de energia, duração e quantidade de ressacas para as fases isoladas e combinadas de cada modo e o valor crítico tabelado de $\mathrm{Chi}^{2}$, para um nível de confiança de $95 \%$. Os resultados apontam valores de $\mathrm{Chi}^{2}$ calculados acima do limite tabelado, tanto para as fases isoladas quanto combinadas, exceto para duração e quantidade das ressacas durante as fases do SAM. Desta forma, rejeitou-se a 
hipótese nula de que a energia, duração e a quantidade de ressacas são estatisticamente independentes das fases dos modos climáticos isolados e combinados, com exceção da duração e quantidade de ressacas em relação as fases do SAM.

Em relação aos valores acumulados dos parâmetros que apresentaram dependência estatística com as fases dos modos entre os anos de 1948 e 2008 tem-se que durante a fase negativa do SAM a energia das ressacas foi maior quando comparada a fase positiva (Tabela 4). Para o ENSO, todos os parâmetros apresentaram maiores valores durante a fase neutra e menores valores durante a fase positiva. Ao considerar as combinações entre as fases dos modos, observou-se que a combinação SAM positivo e ENSO neutro apresentou os maiores valores para todos os parâmetros de ressacas. SAM e ENSO negativos apresentaram os segundos maiores acumulados de energia e duração, já a segunda maior ocorrência acumulada se deu durante a combinação SAM negativos e ENSO neutro. Os menores valores acumulados de energia, duração e quantidade de ressacas se encontraram na combinação entre SAM e ENSO positivos. Na distribuição sazonal das ressacas, observou-se que somente no verão as ressacas tenderam a ocorrer associadas à combinação de ENSO negativos e SAM positivo.

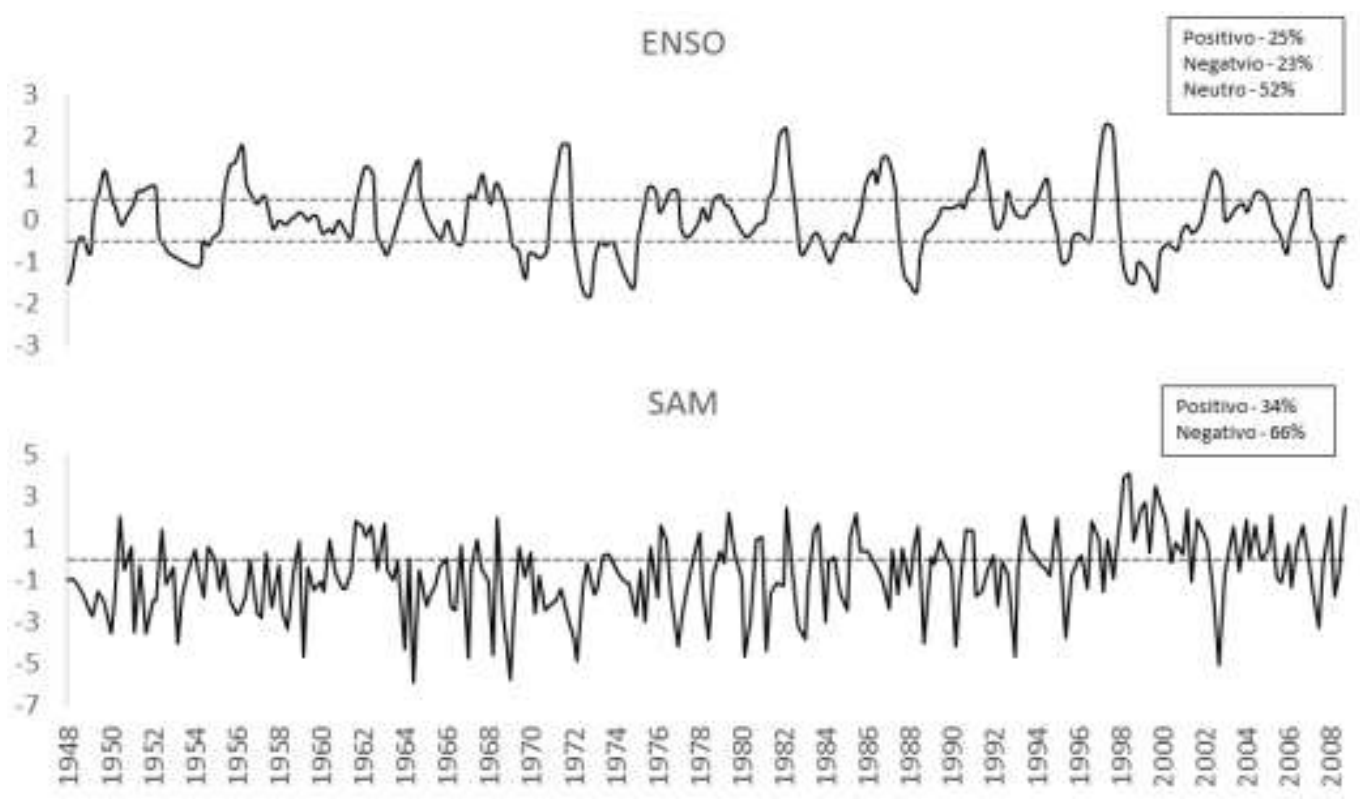

Figura 5 - Série temporal dos índices ENSO (superior) e SAM (inferior) entre os anos de 1948 e 2008 (linhas sólidas). A linha tracejada representa os valores limites entre as fases positivas e negativas do ENSO $(-0,5$ e 0,5$)$ e SAM (0).

Tabela 3 - Valores de Chi $^{2}$ crítico tabelado para nível de confiança de $95 \%$ e calculados com base nos valores acumulados de energia, duração e quantidades para cada fase dos modos de maneira isolada e combinada.

\begin{tabular}{lcccc} 
Chi $^{\mathbf{2}}$ & & & & \\
\hline Tabelado $(\mathrm{P}=0,05)$ & Modo & Energia & Duração & Quantidade \\
3,841 & SAM & 6,833 & 3,865 & 2,769 \\
5,991 & ENSO & 102,341 & 41,177 & 20,966 \\
11,07 & Combinações & 402,477 & 167,33 & 74,177 \\
\hline
\end{tabular}


Tabela 4 - Valores acumulados de energia, duração e quantidade de ressacas entre 1948 e 2008 para cada fase do SAM e ENSO isoladas e combinadas. *valores cujo Chi ${ }^{2}$ calculado ficou abaixo ou muito próximo do valor tabelado e portando, são independentes da fase do modo climático.

\section{SAM}

\section{Negativo Positivo}

Energia $\left(\mathrm{J} / \mathrm{m}^{2}\right)$

521

440

Duração (Dias) *

227

187

Quantidade ( $n^{\circ}$ de eventos) * 116

92

\begin{tabular}{llll} 
ENSO & Negativo & Neutro & Positivo \\
\hline Energia (J/m²) & 313 & 442 & 189 \\
Duração (Dias) & 129 & 191 & 86 \\
Quantidade (n' de eventos) & 61 & 97 & 45
\end{tabular}

\begin{tabular}{|c|c|c|c|c|c|c|}
\hline \multirow{2}{*}{ Combinações } & \multicolumn{3}{|l|}{ SAM+ } & \multicolumn{3}{|l|}{ SAM- } \\
\hline & ENSO- & ENSOn & ENSO+ & ENSO- & ENSOn & ENSO+ \\
\hline Energia $\left(\mathrm{J} / \mathrm{m}^{2}\right)$ & 268 & 553 & 58 & 357 & 332 & 319 \\
\hline Duração (Dias) & 110 & 236 & 28 & 148 & 146 & 144 \\
\hline Quantidade ( $\mathrm{n}^{\circ}$ de eventos) & 56 & 112 & 16 & 66 & 82 & 74 \\
\hline
\end{tabular}

\section{DISCUSSÕES}

\section{CLIMATOLOGIA DAS RESSACAS}

O aumento no número de ressacas entre abril e setembro, com ondas provenientes dos quadrantes sul/sudeste e duração média de dois dias foi também destacado em estudos para a costa do litoral do Rio de Janeiro (BULHÕES ET AL., 2014; DE SOUZA ET AL., 2016; LINS-DE-BARROS ET AL., 2018). Esta distribuição sazonal reflete a passagem de frentes frias provenientes do sul da bacia do Oceano Atlântico, que se dissipam enquanto rumam para nordeste, em direção ao continente Africano (GODOI ET AL 2014; CARDOZO ET AL 2015; PIANCA ET AL, 2010). Segundo Cavalcanti e Kousky (2009), embora estas frentes ocorram durante todo ano, são mais frequentes de maio a outubro, entre $25^{\circ} \mathrm{S}$ e $30^{\circ} \mathrm{S}$, e mais raras ao norte dos $20^{\circ} \mathrm{S}$ no verão.

A maior energia de onda acumulada encontrada durante os meses de junho a agosto é corroborada por Simmonds et al. (2003), que verificaram ao redor da Antártica maior densidade de ciclones no inverno em comparação ao verão. Pianca et al. (2010) discutem o aumento de alturas de ondas durante o inverno e primavera como produto do avanço de frentes polares e da intensificação dos ventos associados a passagem de frentes frias (NIMER, 1989; GAN E RAO, 1991). O aumento do número de ressacas detectadas no presente trabalho durante a primavera encontra-se de acordo com estudos como Andrade (2008) e Calvalcanti e Kuosky (2009), que apontam um aumento do número de frentes frias nesta estação. A menor recorrência de ressacas durante o verão encontra-se em acordo com Nogueira (2014), que apresenta baixos valores de alturas significativas de ondas para a bacia do Espírito Santo nesta estação. Segundo Nogueira (2014), no verão existe uma forte estabilidade atmosférica em decorrência do ASAS, configurando o padrão de ondas com menores alturas. 
A duração média das ressacas deste estudo coincide com o tempo de vida médio de um ciclone subtropical ou extratropical ( $\sim 3$ dias) conforme mostram Gozzo et al. (2014), através da reanálise de dados de pressão atmosférica do modelo ERA-Interim para a porção sudoeste da bacia do oceano Atlântico Sul. Os autores apontam ainda que a duração máxima de um ciclone pode chegar a 7 dias, o que corrobora os resultados encontrados neste estudo. Ressacas de longa duração ( 7 dias) também foram detectadas por Godoi et al. (2014) no litoral do Rio de Janeiro, utilizando reanálise de ondas proveniente do modelo WaveWatchIII.

\section{EFEITOS DOS MODOS DE VARIABILIDADE CLIMÁTICO}

A maior ocorrência de ressacas relacionada ao ENSO neutro se mostra em concordância com o estudo de Beu e Ambrizzi (2006), que destacam a maior atividade de ciclones durante esta fase do ENSO. Os autores ressaltam ainda que há mais anos neutros do que positivos e negativos, assim como encontrado no presente estudo. Porém, os resultados mostram que a fase neutra do ENSO ocorre duas vezes mais do que as outras, enquanto em média, os valores de energia, duração e quantidade acumulados de ressacas na fase neutra do ENSO são 0,5 e 1,2 vezes maiores do que nas fases negativa e positiva respectivamente. Assim, pode-se afirmar que a fase negativa do ENSO possui um alto potencial em termos de energia, duração e quantidade de ressacas, em acordo com Gan e Rao (1991); Rao et al. (2002); Pezza e Ambrizzi (2003); Da Silveira Pereira e Klumb-Oliveira (2015), que apontam maior ciclogênese no Atlântico subtropical próximo ao Uruguai, Argentina e Brasil durante ENSOs negativos. De acordo com Solman e Menéndez (2002), o aumento da baroclinicidade do escoamento básico e da irradiação de energia para níveis mais alto podem estar associados intensificação de ciclones durante o ENSO negativo. Além disso, a fase negativa do ENSO se associa com o enfraquecimento do ASAS e dos ventos alísios, reduzindo o bloqueio para as frentes frias (ARAGÃO, 1998; RAO ET AL., 2002; PEZZA E AMBRIZZI, 2003; SILVA ET AL., 2009).

Reboita e Ambrizzi (2006) e Reboita et al. (2009) descrevem o favorecimento da atividade ciclogenética em médias latitudes ( $\sim 45^{\circ} \mathrm{S}$ ) durante a fase negativa do SAM, em acordo com os valores de energia de ressacas apresentados. Em relação a sazonalidade da ocorrência de ressacas, a preferência de eventos associados à fase negativa do SAM durante o inverno e primavera, e positiva durante o verão, se encontra em concordância com o padrão geral de frentes frias na América do Sul apresentado por Cardozo et al. (2015).

A ocorrência combinada da fase negativa do ENSO com a fase positiva do SAM durante o verão também foi relatada em estudos como Fogt e Bromwich (2006), L'Heureux e Thompson (2006), Hendon et al. (2007) e Marshall et al. (2012). L'Heureux e Thompson (2006) mostram que no verão, aproximadamente $25 \%$ da variabilidade interanual do SAM se encontra linearmente correlacionada com variações do ENSO com índices de nível de confiança acima de $99 \%$. Apesar do verão apresentar baixa ocorrência de ondas típicas de ressacas (PIANCA ET AL. 2010; NOGUEIRA, 2014), a combinação dos modos está associada ao cenário de formação de ciclones em latitudes próximas ao sudeste do Brasil, devido ao tripolo do SAM positivo (REBOITA ET AL., 2014) 
e enfraquecimento do ASAS por efeito do ENSO negativo (RAO ET AL., 2002; PEZZA E AMBRIZZI, 2003; SILVA ET AL., 2009), o que acarreta na maior ocorrência de ressacas com extremos de alta energia, conforme mostram os resultados.

O mecanismo de intensificação de clicogêneses associado a fase positiva do SAM se mostra em acordo com os maiores valores de energia, duração e quantidade de ressacas durante a combinação com a fase neutra do ENSO. Porém, perde seu potencial quando combinado à fase positiva do ENSO, que promove o fortalecimento do ASAS devido à intensificação da subsidência de ar nas regiões tropicais no Atlântico Sul (KOUSKY et al., 1984) e intensifica os ventos alísios no Brasil obstruindo de frentes frias (ARAGÃO, 1998), corroborando com os resultados de redução drástica da energia, duração e quantidade das ressacas.

\section{CONCLUSÕES}

A utilização de dados de ondas modelados se mostrou uma alternativa adequada à falta de dados medidos e uma ferramenta eficaz na caracterização do clima de ressacas, haja visto a boa concordância com estudos atmosféricos pretéritos. Este estudo faz parte de um esforço para aumentar a quantidade de informações sobre a influência de padrões climáticos globais sobre o clima de ressacas na bacia do Oceano Atlântico Sul.

Com base no teste de hipótese $\mathrm{Chi}^{2}$ a um nível de confiança de $95 \%$, os parâmetros de ressacas se mostraram estatisticamente dependentes das fases dos modos climáticos isolados e combinados, com exceção da duração e quantidade de ressacas em relação a fase do SAM.

O padrão de variação de energia, duração e quantidade de ressacas, em relação aos modos climáticos, se mostrou em acordo com a literatura prévia. De maneira isolada, as fases negativas do SAM e neutra do ENSO estiveram associadas às maiores energias, durações e quantidades de ressacas, embora, proporcionalmente, a fase negativa do ENSO apresente valores acumulados altos dos parâmetros de ressacas. Já os menores valores acumulados encontraram-se nas fases positivas de SAM e ENSO.

Em relação à combinação entre os modos climáticos, os maiores valores de parâmetros ocorreram em períodos de SAM positivo concomitante ao ENSO neutro. Enquanto as menores energias, durações e quantidades acumuladas se deram na combinação entre SAM e ENSO positivos, ressaltando a importância de avaliar o impacto da combinação dos modos.

Conclui-se, com base nos 60 anos dados de ondas do modelo GOW, que temporada de ressacas no litoral sul do Espírito tem seu auge entre os meses de julho e setembro e foi caracterizada por eventos duradouros e energéticos que evoluem para eventos breves e mais frequentes. Em função da proveniência do quadrante sudeste e da duração média de dois dias, a ondas de ressacas podem ser associadas a entradas de frentes frias. 


\section{AGRADECIMENTOS}

Os autores deste trabalho agradecem ao Auxílio no 88887.145855/201700 da Coordenação de Aperfeiçoamento de Pessoal de Nível Superior (CAPES) e ao Processo Universal 421657/2016-2 do Conselho Nacional de Desenvolvimento Científico e Tecnológico (CNPq).

O conjunto de dados do Projeto de Reanálise 20CRV2c foi fornecido pelo Departamento de Energia dos EUA, pelo Escritório de Ciência Inovador, pelo Programa de Impacto Computacional na Teoria e Experimentação (DOE INCITE), pelo Escritório de Pesquisa Biológica e Ambiental (BER) e pelo Administração nacional oceânica e atmosférica dos EUA (National Oceanographic and Atmospheric Administration - NOAA).

O conjunto de dados Índice Niño Oceânico (Oceanic Niño Index - ONI) foi fornecido pelo Serviço Nacional de previsão do tempo do EUA, Centro Nacional de Previsão Climática e pela Administração nacional oceânica e atmosférica dos EUA (National Oceanographic and Atmospheric Administration - NOAA).

À Dra. Eliana Zandonate do Departamento de Estatística da Universidade Federal do Espírito Santo pelo apoio e orientação na aplicação dos testes estatísticos.

\section{REFERÊNCIAS BIBLIOGRÁFICAS}

ALBINO, J., NETO, N. C., \& OLIVEIRA, T. C. A. (2016a). The Beaches of Espírito Santo. In Brazilian Beach Systems (pp. 333-361). Springer, Cham.

ALBINO, J., JIMÉNEZ, J. A., \& OLIVEIRA, T. C. (2016b). Planform and mobility in the Meaípe-Maimbá embayed beach on the South East coast of Brazil. Geomorphology, 253, 110-122.

AMARANTE, O.A.C., SILVA, F.J.L., ANDRADE, P.E.P, PARECY, E. (2009) Atlas eólico: Espírito Santo. Agência de Serviços Públicos de Energia do Estado do Espírito Santo (ASPE), Vitória.

ANDRADE, K. (2009). Climatologia e comportamento dos sistemas frontais sobre a América do Sul. 2007. 187 f. Dissertação de Mestrado em Meteorologia - Instituto Nacional de Pesquisas Espaciais, São José dos Campos.

ARAGÃO, J.O.R. (1998) - O Impacto do ENSO e do Dipolo do Atlântico no Nordeste do Brasil. Bulletin de I'Institut Français d'Etudes Andines, 27(3):839844. DOI: $10.1590 / \mathrm{S} 1415-43662009000400014$

BEU, C. M. L., \& AMBRIZZI, T. (2006). Variabilidade interanual e intersazonal de frequencia de ciclones no Hemisfério Sul. Rev. Bras. Meteorol, 21, 44-55.

BULHÕES, E. M. R., FERNANDEZ, G. B., DE OLIVEIRA FILHO, S. R., PEREIRA, T. G., \& DA ROCHA, T. B. (2014). Impactos costeiros induzidos por ondas de tempestade entre o Cabo Frio e o Cabo Búzios, Rio de Janeiro, Brasil. Quaternary and Environmental Geosciences, 5(2).

CARDOZO, A. B., CUSTÓdio, I. S., REBOITA, M. S., \& GARCIA, S. R. (2015). Climatologia de frentes frias na américa do sul e sua relação com o modo anular sul. Revista Brasileira de Climatologia, 17. 
CARLSON, T. N. (1991), Mid-Latitude Weather Systems, 507 pp., Am.Meteorol. Soc., Boston, Mass.

CASTELLE, B., BUJAN, S., FERREIRA, S., \& DODET, G. (2017). Foredune morphological changes and beach recovery from the extreme 2013/2014 winter at a high-energy sandy coast. Marine Geology, 385, 41-55.

CAVALCANTI, I. F. A., \& KOUSKY, V. E. (2009). Frentes frias sobre o Brasil. Tempo e clima no Brasil. São Paulo: Oficina de Textos, 135-47.

CHANG, E. K., LEE, S., \& SWANSON, K. L. (2002). Storm track dynamics. Journal of climate, 15(16), 2163-2183.

COWELL, P. J., \& THOM, B. G. (1994). Morphodynamics of coastal evolution (pp. 33-86). Cambridge University Press, Cambridge, United Kingdom and New York, NY, USA.

DA SILVEIRA PEREIRA, N. E., \& KLUMB-OLIVEIRA, L. A. (2015). Analysis of the influence of ENSO phenomena on wave climate on the central coastal zone of Rio de Janeiro (Brazil). Revista de Gestão Costeira Integrada-Journal of Integrated Coastal Zone Management, 15(3), 353-370.

DE SOUZA, T. A., BULHÕES, E., \& DA SILVA AMORIM, I. B. (2016). Ondas de tempestade na costa Norte Fluminense. Quaternary and Environmental Geosciences, 6(2).

DIRETORIA DE HIDROGRAFIA E NAVEGAÇÃO. CENTRO DE HIDROGRAFIA DA MARINHA. Banco nacional de dados oceanográficos. Previsões de maré. Disponível em http://www.mar.mil.br/dhn/chm/box-previsaomare/tabuas/40280Jan2017.htm. Acesso em: 07 de janeiro de 2020.

DISSANAYAKE, P. M. P. K., BROWN, J., \& KARUNARATHNA, H. (2015). Impacts of storm chronology on the morphological changes of the Formby beach and dune system, UK. Natural Hazards and Earth System Science, 15(7), 15331543.

DURÁN, R., GUILLÉN, J., RUIZ, A., JIMÉNEZ, J. A., \& SAGRISTÀ, E. (2016). Morphological changes, beach inundation and overwash caused by an extreme storm on a low-lying embayed beach bounded by a dune system (NW Mediterranean). Geomorphology, 274, 129-142.

EARLIE, C., MASSELINK, G., \& RUSSELL, P. (2018). The role of beach morphology on coastal cliff erosion under extreme waves. Earth Surface Processes and Landforms, 43(6), 1213-1228.

FOGT, R. L., \& BROMWICH, D. H. (2006). Decadal variability of the ENSO teleconnection to the high-latitude South Pacific governed by coupling with the southern annular mode. Journal of Climate, 19(6), 979-997.

GAN, M. A., \& RAO, V. B. (1991). Surface cyclogenesis over South America. Monthly Weather Review, 119(5), 1293-1302.

GODOI, V. A., BRYAN, K. R., \& GORMAN, R. M. (2018). Storm wave clustering around New Zealand and its connection to climatic patterns. International Journal of Climatology, 38, e401-e417. 
GODOI, V. A., RIBEIRO, C. E. P., \& TORRES JR, A. R. (2014). An overview of events of high sea waves at the mouth of Guanabara Bay Pan-American. J Aquat Sci, 9(2), 70-87.

GONG, T., FELDSTEIN, S. B., \& LUO, D. (2010). The impact of ENSO on wave breaking and southern annular mode events. Journal of the atmospheric sciences, 67(9), 2854-2870.

GOTELLI, N. J., \& ELLISON, A. M. (2016). Princípios de estatística em ecologia. Artmed Editora.

GOZZO, L. F., DA ROCHA, R. P., REBOITA, M. S., \& SUGAHARA, S. (2014). Subtropical cyclones over the southwestern South Atlantic: Climatological aspects and case study. Journal of Climate, 27(22), 8543-8562.

HAMMER, Ø. (2012). PAST PAleontological STatistics Version 2.17 Reference Manual. Natural History Museum, university of oslo, 229.

HAMMER, Ø., HARPER, D. A., RYAN, P. D. (2001) - PAST: Paleontological statistics software package for education and data analysis. Palaeontologia electronica, 4(1), 9.

HENDON, H. H., THOMPSON, D. W., \& WHEELER, M. C. (2007). Australian rainfall and surface temperature variations associated with the Southern Hemisphere annular mode. Journal of Climate, 20(11), 2452-2467.

HOLTHUIJSEN, L. H. (2007). Linear wave theory (oceanic waters). Waves in oceanic and coastal waters, 106-142.

HUANG, B., THORNE, P. W., BANZON, V. F., BOYER, T., CHEPURIN, G., LAWRIMORE, J. H., MENNE, M. J., SMITH, T. M., VOSE, R. S. \& ZHANG, H. M. (2017). Extended reconstructed sea surface temperature, version 5 (ERSSTv5): upgrades, validations, and intercomparisons. Journal of Climate, 30(20), 81798205.

INNOCENTINI, V., ARANTES, F. O., \& PRADO, S. C. C. (2003). Modelo de ondas aplicado ao caso 5-8 de maio de 2001. Revista Brasileira de Meteorologia, 18(01), 97-104.

KOUSKY, V. E., KAGANO, M. T., \& CAVALCANTI, I. F. (1984). A review of the Southern Oscillation: oceanic-atmospheric circulation changes and related rainfall anomalies. Tellus $A, 36(5), 490-504$.

KOUSKY, V. E., KAGANO, M. T., \& CAVALCANTI, I. F. (1984). A review of the Southern Oscillation: oceanic-atmospheric circulation changes and related rainfall anomalies. Tellus $A, 36(5), 490-504$.

L'HEUREUX, M. L., \& THOMPSON, D. W. (2006). Observed relationships between the El Niño-Southern Oscillation and the extratropical zonal-mean circulation. Journal of Climate, 19(2), 276-287.

LARKIN, N.K.; HARRISON, D.E. (2005) - On the definition of El Niño and associated seasonal average U.S. weather anomalies. Geophysical Research Letters, 31(L12705):1-4. DOI: 10.1029/2005GL022738.

LINS-DE-BARROS, F. M., KLUMB-OLIVEIRA, L., \& DE FRANÇA LIMA, R. (2018). Avaliação histórica da ocorrência de ressacas marinhas e danos associados entre os anos de 1979 e 2013 no litoral do estado do Rio de Janeiro (Brasil). Journal 
of Integrated Coastal Zone Management/Revista de Gestão Costeira Integrada, 18(2), 85-102.

LORENZ, D. J., \& HARTMANN, D. L. (2001). Eddy-zonal flow feedback in the Southern Hemisphere. Journal of the atmospheric sciences, 58(21), 3312-3327.

LÜBBECKE, J. F., BURLS, N. J., REASON, C. J., \& MCPHADEN, M. J. (2014). Variability in the South Atlantic anticyclone and the Atlantic Niño mode. Journal of Climate, 27(21), 8135-8150.

MARSHALL, A. G., HEMER, M. A., HENDON, H. H., \& MCINNES, K. L. (2018). Southern annular mode impacts on global ocean surface waves. Ocean Modelling, 129, 58-74.

MARSHALL, A. G., HUDSON, D., WHEELER, M. C., HENDON, H. H., \& ALVES, O. (2012). Simulation and prediction of the Southern Annular Mode and its influence on Australian intra-seasonal climate in POAMA. Climate dynamics, 38(11-12), 2483-2502.

MASSELINK, G., HUGHES, M., \& KNIGHT, J. (2014). Introduction to coastal processes and geomorphology. Routledge.

MENDOZA, E. T., \& JIMÉNEZ, J. A. (2009). Regional vulnerability analysis of Catalan beaches to storms. In Proceedings of the Institution of Civil EngineersMaritime Engineering (Vol. 162, No. 3, pp. 127-135). Thomas Telford Ltd.

NIMER, E. (1989). Climatologia do brasil. IBGE.

NOGUEIRA, I. C. M. (2014). Caracterização do clima de ondas na bacia do espírito santo através de modelagem numérica. Dissertação (Mestrado em Engenharia Oceânica) Universidade Federal de Rio de Janeiro/COPPE. p. 128.

PARISE, C. K., CALLIARI, L. J., \& KRUSCHE, N. (2009). Extreme storm surges in the south of Brazil: atmospheric conditions and shore erosion. Brazilian Journal of Oceanography, 57(3), 175-188.

PEZZA, A. B., \& AMBRIZZI, T. (2003). Variability of Southern Hemisphere cyclone and anticyclone behavior: Further analysis. Journal of Climate, 16(7), 1075-1083.

PHYSICAL SCIECES DIVISION. Earth System Research Laboratory. National Oceanic and Atmospheric Administration. 20CR Climate Indices: Southern Annular Mode. Disponível em: https://www.esrl.noaa.gov/psd/data/20thC_Rean/timeseries/monthly/SAM/. Acesso em 10 de novembro de 2019.

PIANCA, C., MAZZINI, P. L. F., \& SIEGLE, E. (2010). Brazilian offshore wave climate based on NWW3 reanalysis. Brazilian Journal of Oceanography, 58(1), 53-70.

RAHMSTORF, S. (2017). Rising hazard of storm-surge flooding. Proceedings of the National Academy of Sciences, 114(45), 11806-11808.

RAO, V. B., DO CARMO, A. M. C., \& FRANCHITO, S. H. (2002). Seasonal variations in the Southern Hemisphere storm tracks and associated wave propagation. Journal of the atmospheric sciences, 59(6), 1029-1040. 
REBOITA, M. S., ROCHA, R. D., \& AMBRIZZI, T. (2005). Climatologia de ciclones sobre o Atlântico Sul utilizando métodos objetivos na detecção destes sistemas. In CONGRESSO ARGENTINO DE METEOROLOGIA (Vol. 9).

REBOITA, M. S., \& AMBRIZZI, T. (2006). Monitoramento dos Ciclones Extratropicais no Hemisfério Sul. In Congresso Brasileiro de Meteorologia (Vol. 14).

REBOITA, M. S., AMBRIZZI, T., \& ROCHA, R. P. D. (2009). Relationship between the southern annular mode and southern hemisphere atmospheric systems. Revista Brasileira de Meteorologia, 24(1), 48-55.

REBOITA, M. S., DA ROCHA, R. P., AMBRIZZI, T., \& GOUVEIA, C. D. (2014). Trend and teleconnection patterns in the climatology of extratropical cyclones over the Southern Hemisphere. Climate Dynamics, 45(7-8), 1929-1944.

REGUERO, B. G., MENÉNDEZ, M., MÉNDEZ, F. J., MÍNGUEZ, R., \& LOSADA, I. J. (2012). A Global Ocean Wave (GOW) calibrated reanalysis from 1948 onwards. Coastal Engineering, 65, 38-55.

SATYAMURTY, P., \& DE MATTOS, L. F. (1989). Climatological lower tropospheric frontogenesis in the midlatitudes due to horizontal deformation and divergence. Monthly Weather Review, 117(6), 1355-1364.

SILVA, G. A. M., AMBRIZZI, T., \& MARENGO, J. A. (2009). Observational evidences on the modulation of the South American Low Level Jet east of the Andes according the ENSO variability. In Annales geophysicae: atmospheres, hydrospheres and space sciences (Vol. 27, No. 2, p. 645).

SIMMONDS, I., KEAY, K., \& LIM, E. P. (2003). Synoptic activity in the seas around Antarctica. Monthly Weather Review, 131(2), 272-288.

SUN, X., COOK, K. H., \& VIZY, E. K. (2017). The South Atlantic subtropical high: climatology and interannual variability. Journal of Climate, 30(9), 3279-3296.

THOMPSON, D. W., \& WALLACE, J. M. (2000). Annular modes in the extratropical circulation. Part I: Month-to-month variability. Journal of climate, 13(5), 1000-1016.

VENEGAS, S. A., MYSAK, L. A., \& STRAUB, D. N. (1997). Atmosphere-ocean coupled variability in the South Atlantic. Journal of Climate, 10(11), 2904-2920.

VERA, C. S., P. K. VIGLIAROLO, AND E. H. BERBERY (2002), Cold season synoptic- scale waves over subtropical South America, Mon. Weather Rev., 130, 684-699.

YOU, Z. J., \& LORD, D. (2008). Influence of the El Niño-Southern Oscillation on NSW Coastal Storm Severity. Journal of Coastal Research, 24(sp2), 203-207.

ZAR, J. H. (1996). Biostatistical analysis, 3rd edn Prentice Hall International. London 